\title{
Treatment of Diabetic Foot Ulcer Using Matriderm In Comparison with a Skin Graft
}

\author{
Hyojin Jeon, Junhyung Kim, Hyeonjung Yeo, Hoijoon Jeong, Daegu Son, Kihwan Han \\ Department of Plastic and Reconstructive Surgery, Keimyung University School of Medicine, Daegu, Korea
}

Background For patients with neuropathy, vasculopathy, and impairment of wound healing, treatment of a diabetic foot ulcer poses many challenges. A large number of dermal analogues have been invented in an effort to overcome these challenges. Matriderm, a dermal analogue, is made from bovine collagen and elastin. This study was conducted in order to evaluate the effectiveness of Matriderm for treatment of diabetic foot ulcers, in comparison with skin grafting.

Methods Sixty patients with diabetic foot ulcer were included in this prospective study. The average age of the patients, who had type II diabetes mellitus, was 58 years old. The patients were allocated to an experimental or control group with their consents. The patients were selected with their consent for inclusion in an experimental group and a control group. Patients in the experimental group received a Matriderm appliance and a split-thickness skin graft, while those in the control group received only a split-thickness skin graft.

Results A shorter hospitalization period (7.52 weeks) was observed in the experimental group than in the control group (9.22 weeks), and a shorter period of time (8.61 weeks) was required for complete healing, compared with the control group (12.94 weeks), with statistical significance $(\mathrm{P}<0.05)$. A higher elasticity ratio of the affected side to the non-affected side was observed in the experimental group, compared with the control group $(\mathrm{P}<0.01)$.

Conclusions Matriderm enables effective healing and improves elasticity in treatment of patients with diabetic foot ulcer.

Keywords Skin / Diabetes complications / Skin transplantation
Correspondence: Junhyung Kim Department of Plastic and Reconstructive Surgery, Keimyung University School of Medicine, 56 Dalseong-ro, Jung-gu, Daegu 700-712, Korea Tel: $+82-53-250-7635$ Fax: +82-53-255-0632 E-mail: med69@dsmc.or.kr

This article was presented at the 70th Congress of the Korean Society of Plastic and Reconstructive Surgeons on November 9-11, 2012 in Seoul, Korea.

No potential conflict of interest relevant to this article was reported.

Received: 19 Mar 2013 • Revised: 15 May 2013 • Accepted: 20 Jun 2013

pISSN: 2234-6163 • elSSN: 2234-6171 • http://dx.doi.org/10.5999/aps.2013.40.4.403 • Arch Plast Surg 2013;40:403-408

\section{INTRODUCTION}

A person with diabetes may experience one or more of its many complications, such as neuropathy, retinopathy, nephropathy, and arterial occlusive disease. One of the most important clinical manifestations of diabetes is diabetic foot ulcer, the most frequent cause of lower leg amputation [1]. A diabetic foot ulcer has a complicated etiology, and can easily progress to a chronic ulcer. Therefore, a multifactorial approach is needed [2].

Correction of peripheral vasculopathy, adequate debridement, and wound management including infection control are important for treatment of a diabetic foot ulcer. If the diabetic foot ulcer is superficial, a simple wound dressing may be adequate. However, if the ulcer is sufficiently deep, with a layer of subcutaneous fat, along with exposed bone or ligament, a surgical procedure, such as skin graft or flap operation, is needed [3]. A

Copyright ( 2013 The Korean Society of Plastic and Reconstructive Surgeons

This is an Open Access article distributed under the terms of the Creative Commons Attribution Non-Commercial License (http://creativecommons.org/

licenses/by-nc/3.0/) which permits unrestricted non-commercial use, distribution, and reproduction in any medium, provided the original work is properly cited.

www.e-aps.org 
skin graft is a relatively simple procedure, but has limitations, including a high possibility of loss of graft skin and low endurance against friction and pressure. On the other hand, a flap operation is effective and durable for deep ulcers but has a narrow patient spectrum and is associated with severe postoperative complications, including flap necrosis.

The purpose of this study was to investigate the effectiveness of Matriderm (Skin Health, Billerbeck, Germany), an artificial dermis, by comparison with skin graft only in treatment of diabetic foot ulcer $[4,5]$.

\section{METHODS}

\section{Patients}

After receiving institutional review board approval, a prospective study was conducted. From January 2011 to January 2012, 60 patients (male, 37; female, 23) who underwent treatment of diabetic foot ulcer in our department, with a mean age of 58 years (range, 34 to 73 years) and a postoperative follow-up period of 8.8 months (range, 6 to 12 months) were evaluated for enrollment in the study.

Proper wound bed preparation was performed before the study, including debridement (removing all non-viable tissues), controlling infection (based on the results of bacterial culture), and maintaining a balanced moist environment.

Inclusion criteria were type 2 diabetic patients with a chronic foot ulcer, lasting more than four weeks after appropriate management, measuring more than $1 \mathrm{~cm}^{2}$ in size, three sequential negative wound culture tests, and being covered with healthy granulation tissue after proper wound bed preparation. Exclusion criteria were a wound depth of Wagner grade 3, or a University of Texas wound classification greater than 3 , or a wound with vasculopathy of less than 0.9 on the ankle brachial index $(\mathrm{ABI})[6,7]$.

After sufficient explanation of the operation, including the costs of Matriderm, the 60 patients were divided equally into a control group $(n=30)$, receiving a skin graft only, and an experimental group $(n=30)$, receiving the Matriderm appliance combined with a skin graft, with the consent of the patients.

\section{Surgical technique}

In both groups, adequate debridement was performed until healthy tissue was exposed with pin point bleeding. After debridement, Matriderm was applied, soaked with normal saline, and a split-thickness skin graft was performed in one stage in the experimental group (Fig. 1). Only a split-thickness skin graft was performed in the control group (Fig. 2). Wet-to-dry compressive dressing was administered until the graft skin had taken in both groups. After the graft skin had taken, ointment (Connettivina gel, Fidia Farmaceutici Spa., Abano Terme, Italy) and foam dressing (Mepilex, Mölnlycke Health Care, Gothenburg, Sweden) were applied daily.

\section{Assessment}

For comparison of two groups, four factors were measured. 1) The length of the hospital stay; 2) The length of complete wound epithelialization; 3) The number of completely healed patients at postoperative 12 weeks; 4) The elasticity ratio of the affected lesion and the contralateral region at the complete wound epithelialization point, using a skin diagnosis system (Aramo-TS, Aram Huvis Co. Ltd, Sungnam, Korea) [8].

To obtain objective results, the investigator responsible for evaluating the elasticity ratio was blinded. Complete healing was defined as complete wound epithelialization without any

\section{Fig. 1. Experimental group with Matriderm appliance}

(A) Debridement was performed until pin point bleeding and healthy granulation were observed. (B) Matriderm was applied to the wound. (C) Complete hydration of Matriderm was performed. (D) A split-thickness skin graft was performed in one stage.
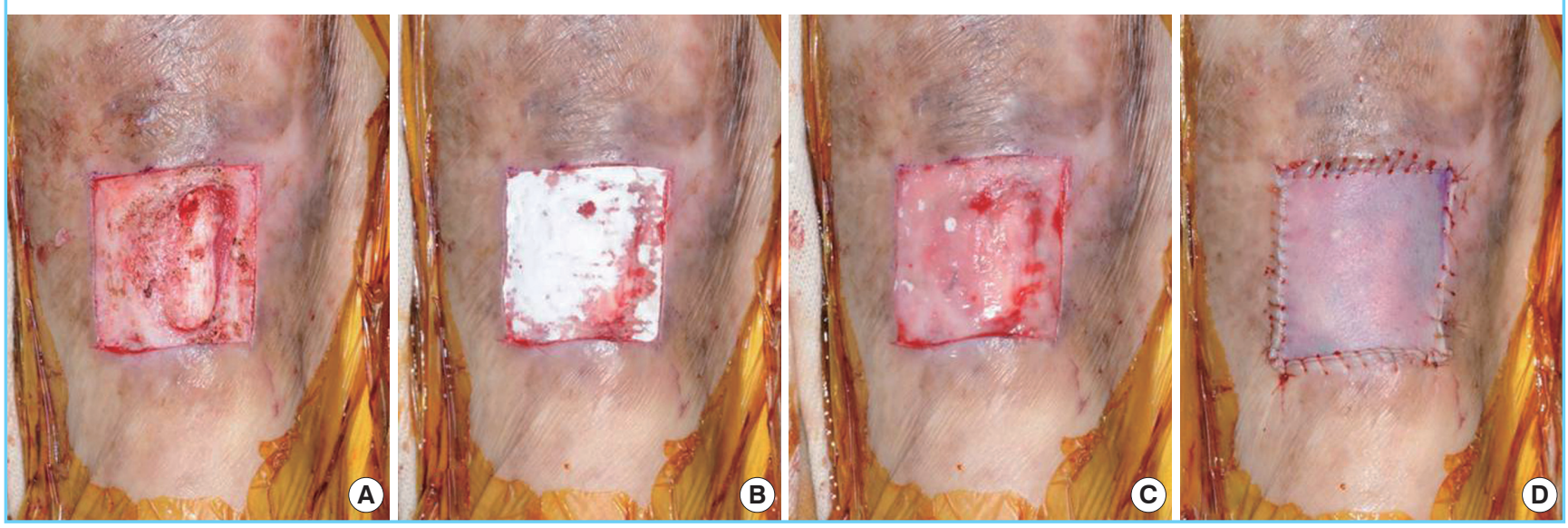


\section{Fig. 2. Control group without Matriderm appliance}

(A) Debridement was performed until pin point bleeding and healthy granulation were observed. Subcutaneous tissue was intact with healthy features. (B) Only a split-thickness skin graft was performed.
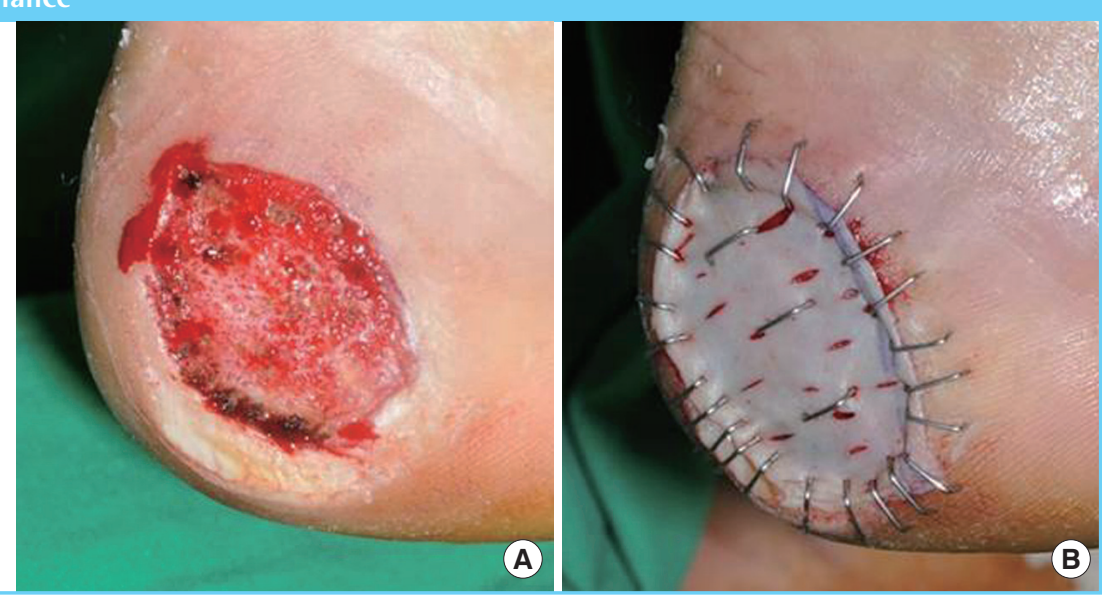

\begin{tabular}{|lccc|}
\hline \multicolumn{4}{|c|}{ Table 1. Clinical characteristics of patients } \\
\hline Characteristic & $\begin{array}{c}\text { Control } \\
\text { group }\end{array}$ & $\begin{array}{c}\text { Experimental } \\
\text { group }\end{array}$ & P-value \\
\hline No. of patients & 30 & 30 & - \\
Sex (male/female) & $18 / 12$ & $19 / 11$ & 0.43 \\
Age (yr) & 59.00 & 58.70 & 0.53 \\
HbA1C (\%) & 7.51 & 7.83 & 0.41 \\
Initial size of ulcer (cm²) & 26.30 & 29.20 & 0.78 \\
Ankle brachial index & 0.98 & 1.05 & 0.83 \\
Wagner grade 1/2 & $4 / 26$ & $5 / 25$ & 0.37 \\
University of Texas wound & $4 / 26$ & $5 / 25$ & 0.37 \\
classification depth 1A/2A & & & \\
\hline
\end{tabular}

transudate or exudate from the wound [9]. SPSS ver. 12.0 (SPSS Inc., Chicago, IL, USA) was used for performance of the statistical analysis. The chi-squared test was used for data analysis. Significance was set at a value of $\mathrm{P} \leq 0.05$.

\section{RESULTS}

In total, 60 patients (100\%) participated in the study without follow-up loss. A summary of the demographic details of the control and experimental groups, including age, $\mathrm{HbAlc}(\%)$, wound size, wound depth, and ABI value, is shown in Table 1. No statistically significant differences in the various characteristics were observed between the groups.

The length of the hospital stay was shorter in the experimental group, at 7.52 weeks, than in the control group (9.22 weeks, $\mathrm{P}<0.05$ ). In addition, the length of wound epithelialization was shorter in the experimental group, at 8.61 weeks, than in the control group (12.94 weeks, $\mathrm{P}<0.05$ ). The number of completely healed patients at postoperative 12 weeks was larger in the experimental group, with 28 patients (93.3\%), than in the control group. With 24 patients $(80.0 \%, \mathrm{P}<0.05)$. The elasticity ratio of the affected lesion and contralateral region at the com-

\begin{tabular}{|lccc|}
\hline Table 2. Postoperative evaluation & & \\
\hline Postoperative evaluation & $\begin{array}{c}\text { Control } \\
\text { group }\end{array}$ & $\begin{array}{c}\text { Experimental } \\
\text { group }\end{array}$ & P-value \\
\hline Hospital stay (wk) & 7.36 & 5.63 & 0.015 \\
Complete healing period (wk) & 8.61 & 12.94 & 0.010 \\
Complete healing rate (\%) & 80.00 & 93.30 & 0.023 \\
Elasticity ratio (affected/ & 0.19 & 0.72 & 0.030 \\
non-affected side) & & & \\
\hline
\end{tabular}

plete wound epithelialization point was higher in the experimental group, at 0.72 , than in the control group $(0.19, \mathrm{P}<0.01)$. A summary of the outcomes of these four factors is shown in Table 2. There was no occurrence of complications, such as a rejection response to the artificial dermis, infection, bleeding problems, or loss of graft skin. Also, during the follow-up period, there was no recurrence in either group.

\section{Case 1}

A 69-year-old male with type 2 diabetes controlled by oral hypoglycemic agents for 11 years suffered from an unhealed diabetic foot ulcer on the tibial side of the right great toe. Debridement was performed primarily, followed by application of Matriderm and a split-thickness skin graft. The patient stayed at the hospital for six weeks and wound epithelialization lasted eight weeks; the elasticity ratio was 0.81 (Fig. 3).

\section{Case 2}

A 34-year-old female with type 2 diabetes controlled by oral hypoglycemic agents for three years suffered from an unhealed diabetic foot ulcer on the right foot dorsum after trauma. Debridement was performed primarily, followed by application of Matriderm and a split-thickness skin graft. The patient stayed in the hospital for five weeks, and wound epithelialization took six weeks; the elasticity ratio was 0.75 (Fig. 4). 


\section{Fig. 3. Case 1 of the experimental group}

A 69-year-old male had a diabetic foot ulcer on the tibial side of the right great toe. The patient stayed in the hospital for six weeks, and had healed completely at eight weeks postoperation. The elasticity ratio measured was 0.81 . (A) After debridement, the periosteum was exposed. (B) Matriderm was applied to the periosteum. (C) A split-thickness skin graft was performed. (D) Image of completely healed state at eight weeks postoperation.
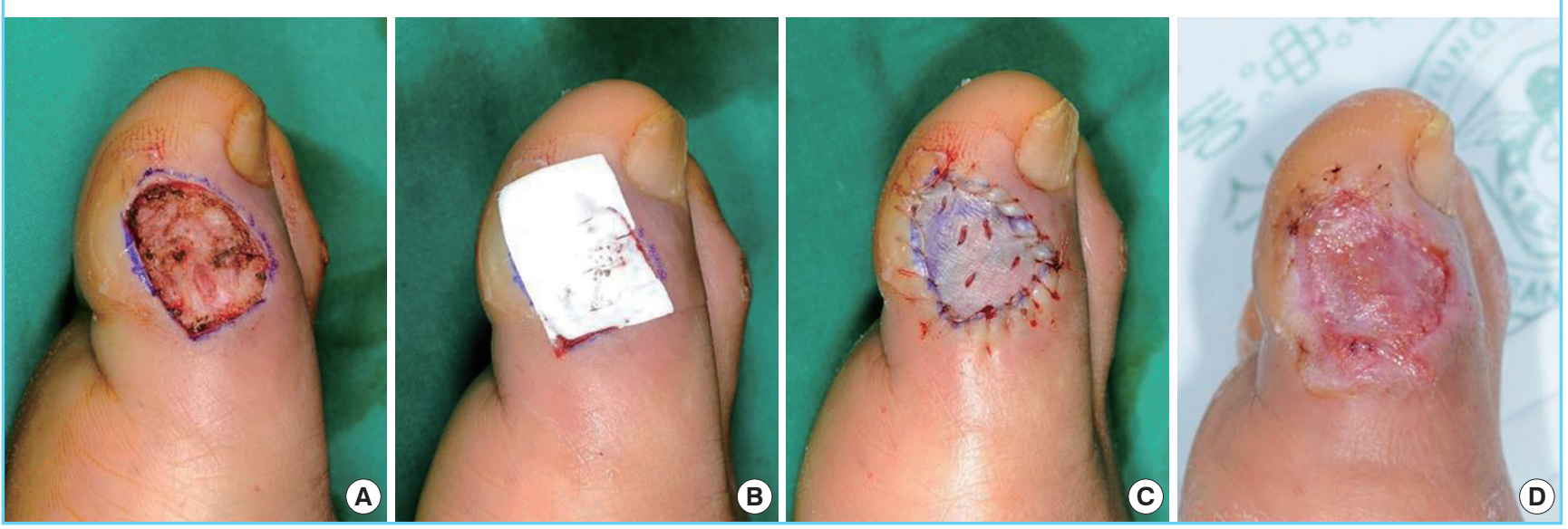

\section{Fig. 4. Case 2 of the experimental group}

A 34-year-old female had a diabetic foot ulcer on the right foot dorsum after trauma. The patient stayed in the hospital for five weeks, and had healed completely at six weeks postoperation. The elasticity ratio was 0.75. (A) After debridement, the fascia was exposed. (B) Matriderm was applied to the fascia. (C) A split-thickness skin graft was performed. Immediate postoperative image. (D) Image of completely healed state at six weeks postoperation.
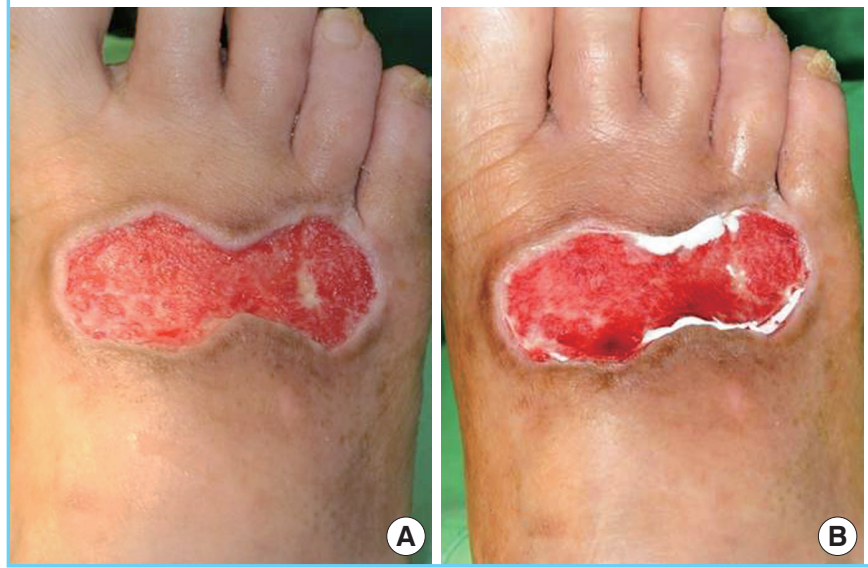

\section{DISCUSSION}

Diabetic foot ulcer is not simply a problem of diabetes complications; it also degrades the quality of life of patients, and can cause fatal complications, such as sepsis. According to the latest statistics for 2003, diabetic foot ulcers occurred in $1.2 \%$ of patients with diabetes foot disease, which is $47.9 \%$ of all cases of foot disease, $54.4 \%$ of all cases of foot amputation $[1,4]$.

In diabetic patients, peripheral circulation disorders due to calcification of the arteries and capillaries, thickening of the basement membrane, diabetic peripheral neuropathy, and hyperglycemia due to lack of growth factors and cell metabolism are causes of impaired wound healing. Therefore, surgical treatment
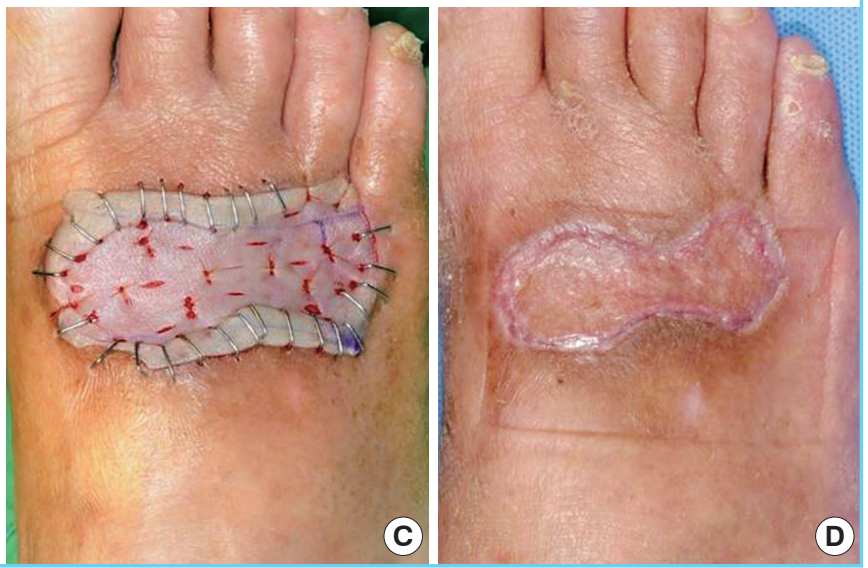

for diabetic foot ulcer with primary closure, skin graft, and flap application are important [2]. Primary closure is possible only for small wounds, and skin grafting is easy to perform; however, the latter does not take well in deep wounds and has low durability. Flap application can overcome the limitations of skin grafting; however, it is burdened with the risk of complications, such as flap necrosis, especially in the case of common diabetes with a poor vascular state.

In the past 10 years, dermis, collagen, and elastic fibers have emerged as important components of wound healing. Cuono et al. [10], a synthesized functional dermal tissue with elastic fiber, has excellent resilience for prevention of scar formation. Matriderm, which was used in our study, is a three-dimensional ma- 


\section{Fig. 5. Meshed Matriderm appliance with poor vascular condition}

The wound after debridement showed poor point bleeding. We assumed that calcification of vessels can cause a midleadingly positive value of ankle brachial index. We applied meshed Matriderm and a meshed split-thickness skin graft in order to facilitate taking of the graft skin.

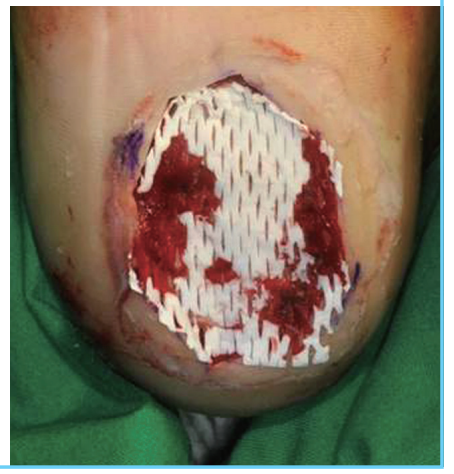

trix composed of collagen fibrils and elastin fibers for support of dermal regeneration in one stage. It induces dermis regeneration as a scaffold, and decreases scar tissue formation. It also provides optimal dermal wound bed preparation as regenerated skin with extensive formation of rete ridges and capillary loops but with the absence of skin appendages and is ready for relatively thinner subsequent skin grafting [11]. Collagen is obtained from the bovine dermis and contains dermal collagen types I, III, and V. Elastin is obtained from the bovine nuchal ligament by hydrolysis. In addition, Matriderm has excellent hemostatic properties and thus reduces the risk of hematoma formation under grafted skin. The non-use of chemical cross-linking of collagen results in biocompatibility $[4,10]$. In our study, the experimental group with Matriderm showed a shorter length of hospital stay and length of complete wound epithelialization and showed a higher elasticity ratio of the affected lesion and contralateral region at the complete wound epithelialization point. We concluded that Matriderm induced dermal regeneration and facilitated the taking of the graft skin. Patients with chronic wounds like diabetic foot ulcers seem to hope for an especially quick return to their daily lives, which was possible in the experimental group.

Although the patients had a good ABI score of 1.05, the wound showed poor pin-point bleeding after debridement (Fig. 5). We assumed that calcification of the vessels can cause a misleadingly good ABI score. We applied a meshed Matriderm and meshed split-thickness skin graft in order to facilitate taking of the graft skin [12]. We expect that meshed Matriderm would also be effective on wounds with a bleeding tendency, such as those associated with liver cirrhosis or chronic renal failure with dialysis. In our study, elasticity was the most important component for data analysis, considering the predictive factors for recurrence $[13,14]$. Although the surgeon and patients were aware of the treatment received, the investigator was blinded and evaluated the elasticity objectively using a skin diagnosis system (Aramo-
TS). The probe for the system can cause constant negative pressure, as much as $300 \mathrm{~mm} \mathrm{Hg}$. By this negative pressure, the sensor of the probe measured the changes in the skin in contact with the probe, and the value of the elasticity was then determined. To take into account the patient's own skin characteristics, we compared both groups according to the elasticity ratio of the affected lesion and contralateral region [15]. As we continue to do follow-up with the patients, we expect lower recurrence in the experimental group, given its higher elasticity, than control group with lower elasticity.

In treatment of diabetic foot ulcer, the experimental group, which received the Matriderm appliance and a split-thickness skin graft, showed a shorter hospital stay, shorter epithelialization period, and higher elasticity ratio than the control group, who received a split-thickness skin graft only. According to these results, we believe that Matriderm can be an effective treatment for diabetic foot ulcer.

\section{REFERENCES}

1. Chung CH, Kim DJ, Kim J, et al. Current status of diabetic foot in Korean patients using national health insurance database. J Korean Diabetes Assoc 2006;30:372-6.

2. Greenhalgh DG. Wound healing and diabetes mellitus. Clin Plast Surg 2003;30:37-45.

3. Kim SH, Kim JW, Kim JB, et al. Multifactorial factors of diabetic foot on diabetes mellitus comparative clinical study. J Korean Soc Plast Reconstr Surg 2002;29:83-90.

4. Hanft JR, Surprenant MS. Healing of chronic foot ulcers in diabetic patients treated with a human fibroblast-derived dermis. J Foot Ankle Surg 2002;41:291-9.

5. Wetzig T, Gebhardt C, Simon JC. New indications for artificial collagen-elastin matrices? Covering exposed tendons. Dermatology 2009;219:272-3.

6. Wagner FWJr. The diabetic foot. Orthopedics 1987;10:16372 .

7. Lavery LA, Armstrong DG, Murdoch DP, et al. Validation of the Infectious Diseases Society of America's diabetic foot infection classification system. Clin Infect Dis 2007;44:5625.

8. Hashmi F, Malone-Lee J. Measurement of skin elasticity on the foot. Skin Res Technol 2007;13:252-8.

9. Eum SJ, Han SK, Gu JH, et al. Treatment of diabetic ulcer using autologous fibroblast-hyaluronic acid complex. J Korean Soc Plast Reconstr Surg 2009;36:548-54.

10. Cuono C, Langdon R, McGuire J. Use of cultured epidermal autografts and dermal allografts as skin replacement after burn injury. Lancet 1986;1:1123-4. 
11. Hamuy R, Kinoshita N, Yoshimoto H, et al. One-stage, simultaneous skin grafting with artificial dermis and basic fibroblast growth factor successfully improves elasticity with maturation of scar formation. Wound Repair Regen 2013; 21:141-54.

12. Han SK, You HJ. Wound coverage using advanced technology in Korea. J Korean Med Assoc 2011;54:594-603.

13. Young MJ, Adams JE, Anderson GF, et al. Medial arterial calcification in the feet of diabetic patients and matched non-diabetic control subjects. Diabetologia 1993;36:61521.

14. Weiner RD, Hlad LM, McKenna DR. Recurrence of diabetic pedal ulcerations following tendo-achilles lengthening. Diabet Foot Ankle 2011;2:6417.

15. Reddy GK. Cross-linking in collagen by nonenzymatic glycation increases the matrix stiffness in rabbit achilles tendon. Exp Diabesity Res 2004;5:143-53. 